Supplement of Hydrol. Earth Syst. Sci., 22, 111-125, 2018

https://doi.org/10.5194/hess-22-111-2018-supplement

(C) Author(s) 2018. This work is distributed under

the Creative Commons Attribution 3.0 License.

(c) (1)

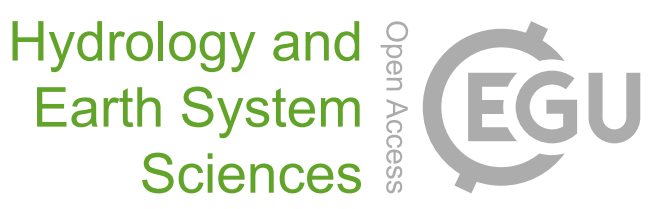

Supplement of

\title{
Pairing FLUXNET sites to validate model representations of land-use/land- cover change
}

\section{Liang Chen et al.}

Correspondence to: Liang Chen (lchen15@gmu.edu)

The copyright of individual parts of the supplement might differ from the CC BY 3.0 License. 
Table S1. Detailed information about the paired sites. Pairs 6, 8, 12 and 16 are obtained from AmeriFlux; the rest of the pairs are from the FLUXNET2015 dataset. The pairs are grouped based on the type of LULCC: pairs 1 5, deciduous broadleaf forest - cropland; pair 6, deciduous broadleaf forest - grassland; pair 7, deciduous broadleaf forest - grassland; pairs $8 \sim 15$, evergreen broadleaf forest grassland; pairs 16 25, evergreen needleleaf forest - open shurbland; pairs 26 27, evergreen needleleaf forest - cropland; pair 28, mixed forest - cropland..

\begin{tabular}{|c|c|c|c|c|c|c|c|}
\hline Pair ID & Name & $\begin{array}{c}\text { Latitude } \\
(\text { degrees N) }\end{array}$ & $\begin{array}{c}\text { Longitude } \\
\text { (degrees E) }\end{array}$ & $\begin{array}{c}\text { Elevation } \\
\text { (m) }\end{array}$ & Land cover type & $\begin{array}{c}\text { Separation } \\
(\mathbf{k m})\end{array}$ & Period \\
\hline \multirow{2}{*}{1} & IT-CA2 & 42.3772 & 12.026 & 200 & Croplands & \multirow{2}{*}{0.36} & \multirow{2}{*}{$2011-2013$} \\
\hline & IT-CA1 & 42.3804 & 12.0266 & 200 & Deciduous Broadleaf Forests & & \\
\hline \multirow{2}{*}{2} & IT-CA2 & 42.3772 & 12.026 & 200 & Croplands & \multirow{2}{*}{0.44} & \multirow{2}{*}{$2011-2013$} \\
\hline & IT-CA3 & 42.38 & 12.0222 & 197 & Deciduous Broadleaf Forests & & \\
\hline \multirow{2}{*}{3} & IT-CA2 & 42.3772 & 12.026 & 200 & Croplands & \multirow{2}{*}{8.75} & \multirow{2}{*}{$2011-2012$} \\
\hline & IT-Ro2 & 42.3903 & 11.9209 & 160 & Deciduous Broadleaf Forests & & \\
\hline \multirow{2}{*}{4} & FR-Gri & 48.8442 & 1.9519 & 125 & Croplands & \multirow{2}{*}{73.3} & \multirow{2}{*}{$2005-2013$} \\
\hline & FR-Fon & 48.4764 & 2.7801 & 90 & Deciduous Broadleaf Forests & & \\
\hline \multirow{2}{*}{5} & BE-Lon & 50.5515 & 4.7461 & 165 & Croplands & \multirow{2}{*}{92.82} & \multirow{2}{*}{$2004-2014$} \\
\hline & BE-Vie & 50.3050 & 5.9980 & 491 & Deciduous Broadleaf Forests & & \\
\hline \multirow{2}{*}{6} & US-Dk1 & 35.9712 & -79.0934 & 168 & Grasslands & \multirow{2}{*}{0.68} & \multirow{2}{*}{$2003-2008$} \\
\hline & US-Dk2 & 35.9736 & -79.1004 & 168 & Deciduous Broadleaf Forests & & \\
\hline \multirow{2}{*}{7} & AU-Rig & -36.6499 & 145.5759 & 152 & Grasslands & \multirow{2}{*}{48.82} & \multirow{2}{*}{$2011-2013$} \\
\hline & AU-Whr & -36.6732 & 145.0294 & 165 & Evergreen Broadleaf Forests & & \\
\hline \multirow{2}{*}{8} & US-Dk1 & 35.9712 & -79.0934 & 168 & Grasslands & \multirow{2}{*}{0.78} & \multirow{2}{*}{$2004-2008$} \\
\hline & US-Dk3 & 35.9782 & -79.0942 & 163 & Evergreen Needleleaf Forests & & \\
\hline \multirow{2}{*}{9} & $\mathrm{CZ}-\mathrm{BK} 2$ & 49.4944 & 18.5429 & 855 & Grasslands & \multirow{2}{*}{0.96} & \multirow{2}{*}{$2004-2006$} \\
\hline & CZ-BK1 & 49.5021 & 18.5369 & 875 & Evergreen Needleleaf Forests & & \\
\hline
\end{tabular}




\begin{tabular}{|c|c|c|c|c|c|c|c|}
\hline \multirow{2}{*}{10} & DE-Gri & 50.9495 & 13.5125 & 385 & Grasslands & \multirow{2}{*}{4.12} & \multirow{2}{*}{$2004-2010$} \\
\hline & DE-Tha & 50.9636 & 13.5669 & 380 & Evergreen Needleleaf Forests & & \\
\hline \multirow{2}{*}{11} & DE-Gri & 50.9495 & 13.5125 & 385 & Grasslands & \multirow{2}{*}{23.49} & \multirow{2}{*}{$2008-2010$} \\
\hline & DE-Obe & 50.7836 & 13.7196 & 735 & Evergreen Needleleaf Forests & & \\
\hline \multirow{2}{*}{12} & US-Fwf & 35.4454 & -111.7718 & 2270 & Grasslands & \multirow{2}{*}{33.91} & \multirow{2}{*}{$2006-2010$} \\
\hline & US-Fmf & 35.1426 & -111.7273 & 546 & Evergreen Needleleaf Forests & & \\
\hline \multirow{2}{*}{13} & NL-Hor & 52.2404 & 5.0713 & 2.2 & Grasslands & \multirow{2}{*}{46.55} & \multirow{2}{*}{$2004-2011$} \\
\hline & NL-Loo & 52.1666 & 5.7436 & 25 & Evergreen Needleleaf Forests & & \\
\hline \multirow{2}{*}{14} & AT-Neu & 47.1167 & 11.3175 & 970 & Grasslands & \multirow{2}{*}{59.57} & \multirow{2}{*}{$2002-2012$} \\
\hline & IT-Ren & 46.5869 & 11.4337 & 1730 & Evergreen Needleleaf Forests & & \\
\hline \multirow{2}{*}{15} & US-Var & 38.4133 & -120.9507 & 129 & Grasslands & \multirow{2}{*}{60.29} & \multirow{2}{*}{$2000-2007$} \\
\hline & US-Blo & 38.8953 & -120.6328 & 1315 & Evergreen Needleleaf Forests & & \\
\hline \multirow{2}{*}{16} & US-NC1 & 35.8118 & -76.7119 & 5 & Open Shrublands & \multirow{2}{*}{4.03} & \multirow{2}{*}{$2005-2005$} \\
\hline & US-NC2 & 35.803 & -76.6685 & 5 & Evergreen Needleleaf Forests & & \\
\hline \multirow{2}{*}{17} & US-Wi6 & 46.6249 & -91.2982 & 371 & Open Shrublands & \multirow{2}{*}{15.27} & \multirow{2}{*}{$2002-2003$} \\
\hline & US-Wi3 & 46.6347 & -91.0987 & 411 & Deciduous Broadleaf Forests & & \\
\hline \multirow{2}{*}{18} & US-Wi6 & 46.6249 & -91.2982 & 371 & Open Shrublands & \multirow{2}{*}{16.22} & \multirow{2}{*}{$2002-2003$} \\
\hline & US-Wi4 & 46.7393 & -91.1663 & 352 & Evergreen Needleleaf Forests & & \\
\hline \multirow{2}{*}{19} & US-Wi6 & 46.6249 & -91.2982 & 371 & Open Shrublands & \multirow{2}{*}{16.57} & \multirow{2}{*}{2002} \\
\hline & US-Wi0 & 46.6188 & -91.0814 & 349 & Evergreen Needleleaf Forests & & \\
\hline \multirow{2}{*}{20} & CA-SF3 & 54.0916 & -106.0053 & 540 & Open Shrublands & 1087 & $2001 \quad 2005$ \\
\hline & CA-SF2 & 54.2539 & -105.8775 & 520 & Evergreen Needleleaf Forests & 19.01 & $2001-2000$ \\
\hline 21 & CA-NS6 & 55.9167 & -98.9644 & 244 & Open Shrublands & 2743 & $2001-2005$ \\
\hline 21 & CA-NS2 & 55.9058 & -98.5247 & 260 & Evergreen Needleleaf Forests & 21.43 & $2001-2005$ \\
\hline 22 & CA-NS6 & 55.9167 & -98.9644 & 244 & Open Shrublands & 3025 & $2001-2005$ \\
\hline 22 & CA-NS1 & 55.8792 & -98.4839 & 260 & Evergreen Needleleaf Forests & 50.25 & $2001-2003$ \\
\hline 23 & CA-NS6 & 55.9167 & -98.9644 & 244 & Open Shrublands & 3048 & $2001-2005$ \\
\hline 25 & CA-NS5 & 55.8631 & -98.485 & 260 & Evergreen Needleleaf Forests & 50.40 & $2001-2000$ \\
\hline
\end{tabular}




\begin{tabular}{|c|c|c|c|c|c|c|c|}
\hline \multirow{2}{*}{24} & CA-NS6 & 55.9167 & -98.9644 & 244 & Open Shrublands & \multirow{2}{*}{36.29} & \multirow{2}{*}{$2001-2005$} \\
\hline & CA-NS3 & 55.9117 & -98.3822 & 260 & Evergreen Needleleaf Forests & & \\
\hline \multirow{2}{*}{25} & CA-SF3 & 54.0916 & -106.0053 & 540 & Open Shrublands & \multirow{2}{*}{45.41} & \multirow{2}{*}{$2003-2006$} \\
\hline & CA-SF1 & 54.485 & -105.8176 & 536 & Evergreen Needleleaf Forests & & \\
\hline \multirow{2}{*}{26} & DE-Kli & 50.8929 & 13.5225 & 480 & Croplands & \multirow{2}{*}{8.46} & \multirow{2}{*}{$2004-2014$} \\
\hline & DE-Tha & 50.9636 & 13.5669 & 380 & Evergreen Needleleaf Forests & & \\
\hline \multirow{2}{*}{27} & DE-Kli & 50.8929 & 13.5225 & 480 & Croplands & \multirow{2}{*}{18.42} & \multirow{2}{*}{$2008-2014$} \\
\hline & DE-Obe & 50.7836 & 13.7196 & 735 & Evergreen Needleleaf Forests & & \\
\hline \multirow{2}{*}{28} & DE-RuS & 50.8659 & 6.4472 & 102.76 & Croplands & \multirow{2}{*}{69.96} & \multirow{2}{*}{$2011-2014$} \\
\hline & BE-Vie & 50.3051 & 5.9981 & 493 & Mixed Forests & & \\
\hline
\end{tabular}


Table S2. Model scheme settings for Noah-MP used in this study.

\begin{tabular}{|c|c|}
\hline model options & scheme used in this study \\
\hline dynamic vegetation & on \\
\hline canopy stomatal resistance & Ball-Berry \\
\hline soil moisture factor for stomatal resistance & SIMGM \\
\hline runoff and groundwater & Monin-Obukhov \\
\hline surface layer drag coefficient $(\mathrm{CH} \& \mathrm{CM})$ & Niu \& Yang 06 \\
\hline supercooled liquid water & Niu \& Yang 06 \\
\hline frozen soil permeability & gap=F(3D,cosz) \\
\hline radiation transfer & CLASS \\
\hline snow surface albedo & Jordan91 \\
\hline rainfall \& snowfall & Noah \\
\hline lower boundary of soil temperature & semi-implicit \\
\hline snow/soil temperature time scheme &
\end{tabular}



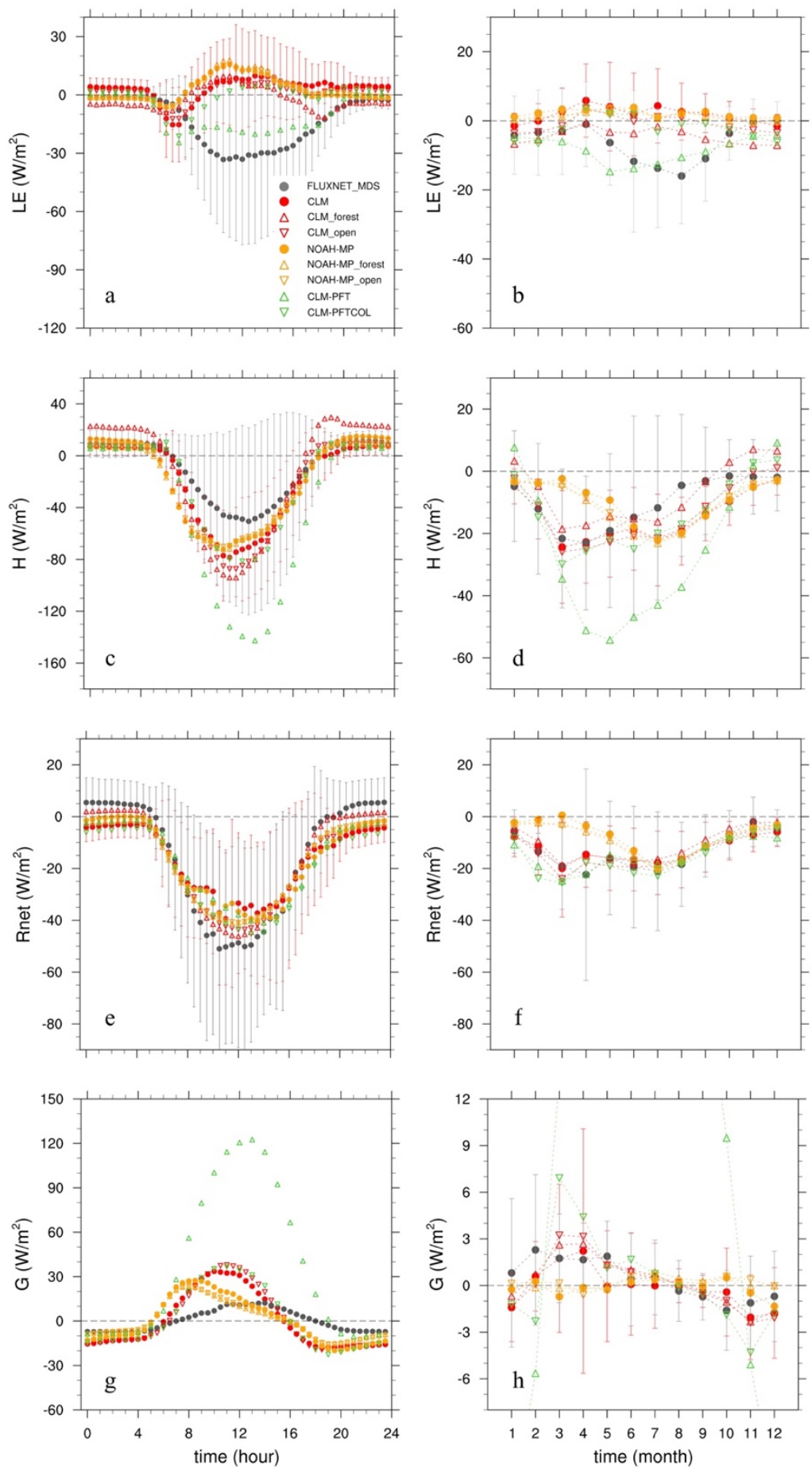

Figure S1. Change in the diurnal (left) and seasonal (right) cycle of $L E\left(\mathrm{~W} / \mathrm{m}^{2}\right.$, a-b), $H\left(\mathrm{~W} / \mathrm{m}^{2}\right.$, cd), $R_{\text {net }}\left(\mathrm{W} / \mathrm{m}^{2}\right.$, e-f), and $G\left(\mathrm{~W} / \mathrm{m}^{2}, \mathrm{~g}-\mathrm{h}\right)$ from forest to open (open - forest) land excluding the pairs $3,7,12$, and 15 . 

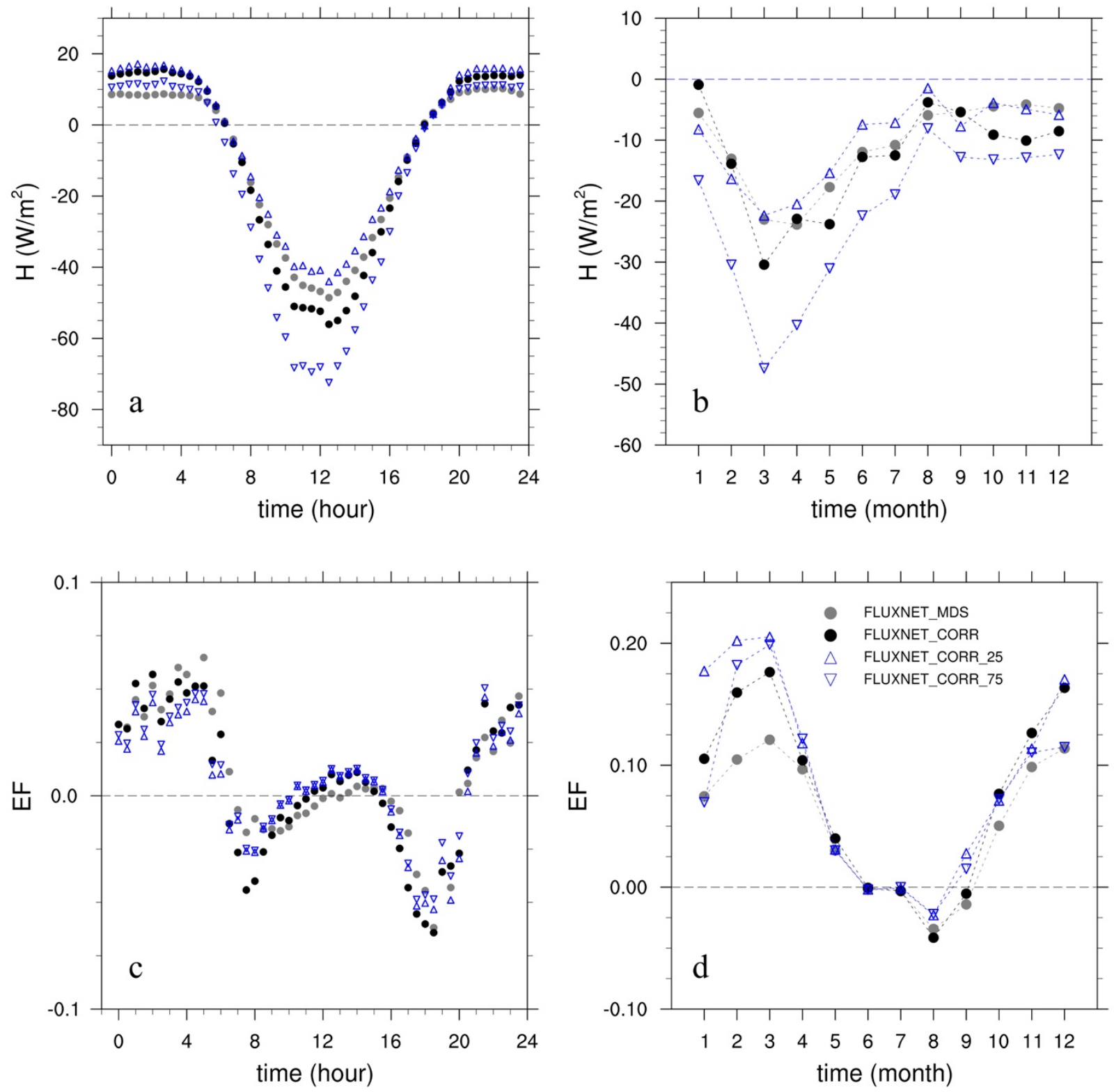

Figure S2. Change in the diurnal (left) and seasonal (right) cycle of $H\left(\mathrm{~W} / \mathrm{m}^{2}\right.$, a-b) and evaporative fraction $(E F, \mathrm{c}-\mathrm{d})$ from forest to open land (open - forest) in the four types of FLUXNET observations. 

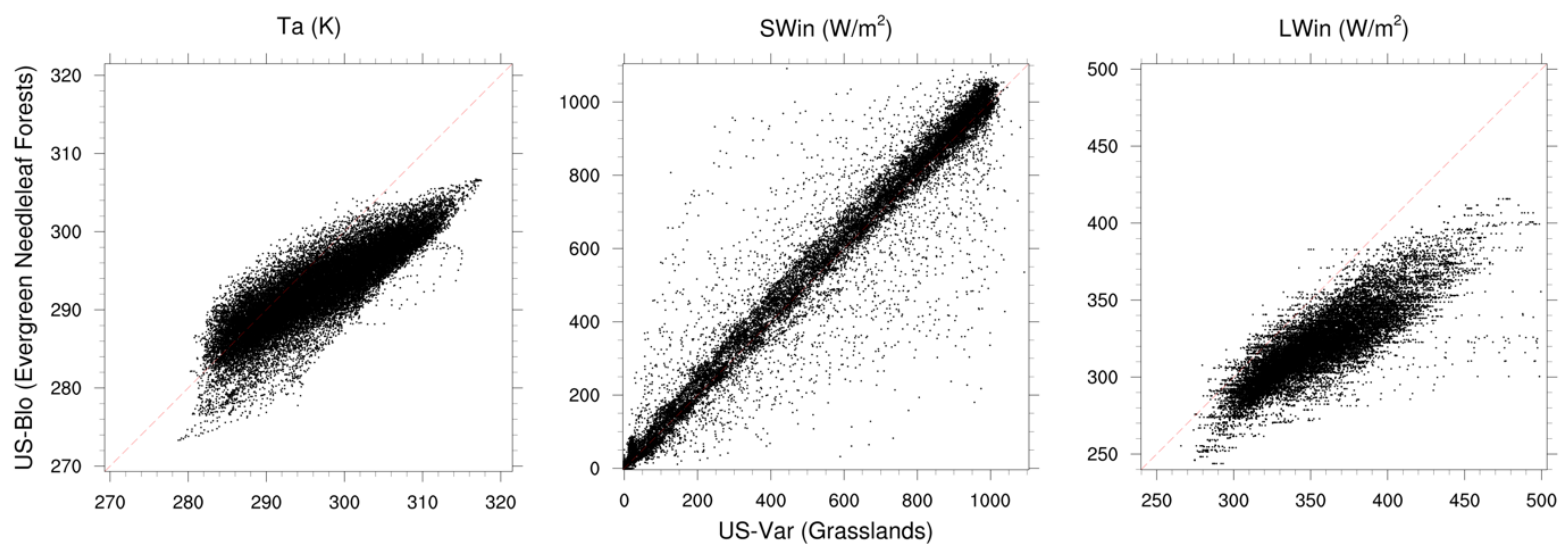

Figure S3. Comparison of air temperature (K, left), downwelling shortwave (middle) and longwave (right) radiations $\left(\mathrm{W} / \mathrm{m}^{2}\right)$ between the paired sites No. 15 . The horizontal axis shows the observations from the grassland site US-Var, and the vertical axis shows the observations from the evergreen needleleaf forest site US-Blo. 


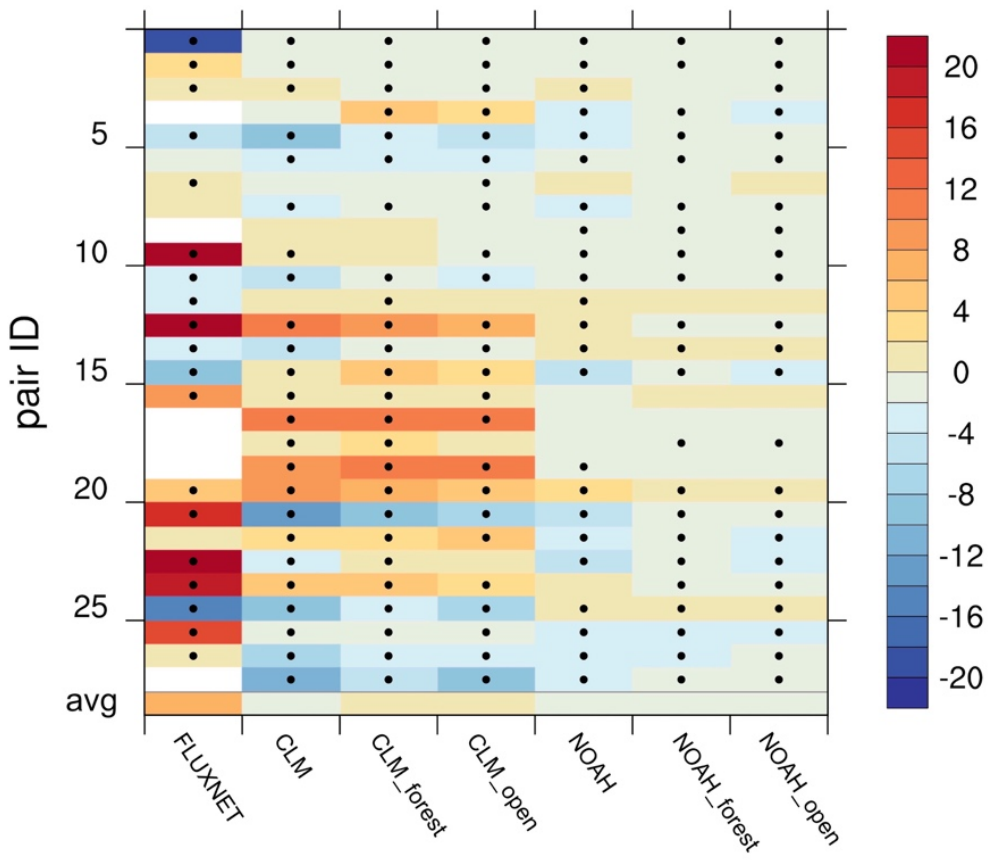

Figure S4. Change (open - forest) in observed and simulated daytime surface soil moisture (\%) during summer (JJA, b). White areas indicate missing observations. 